\title{
Review of: "Validation of methodology for efficient genotyping of CYP2D6 and CYP2C19"
}

\author{
victoria Pratt $^{1}$ \\ 1 Indiana University
}

Potential competing interests: The author(s) declared that no potential competing interests exist.

This manuscript is titled "Validation of methodology for efficient genotyping of CYP2D6 and CYP2C19". Thus this reviewer was expecting a validation with information on analytical sensitivity, analytical specificity, precision, reproducibility, accuracy, and any other parameters associated with analytical validation. Instead this manuscript seems to be a comparison of methodologies on a dataset of samples that were originally genotyped by the Roche Amplichip which has been obsoleted.

major concerns:

Either change the title of the manuscript to better reflect the purpose of the paper or provide the information on analytical validation.

The manuscript spends a significant time on resolving CYP2D6 hybrid samples and trying to determine the nature of the conversion. No single assay in the comparison can completely identify and determine the hybrid conversions. So the authors had to resort to additional methods to resolve. It is not clear if those methods were research or performed in compliance with clinical laboratory standards.

Minor concerns:

Note that Taqman is a trademarked name

Should the Taqman reagents include manufacturer and location in accordance with journal policy? Is there any version for the Agena assay?

Note that there are results in the methods - please remove "with two sample failing quality control...." HGVS nomenclature should be used throughout the manuscript. In particular, 2851C >T and 4181G>C should include the $\mathrm{c}$. 